\title{
昡pubvet
}

https://doi.org/10.31533/pubvet.v14n6a598.1-6

\section{O papel decisivo do meio ácido abomasal na absorção de minerais no trato gastrointestinal de ruminantes}

\author{
Leonardo Marmo Moreira ${ }^{1^{*}}$, Alexandre de Oliveira Teixeira $^{1} \bullet$, Henrique Valentim Nunes \\ Machado $^{10}$, Raphael Pavesi Araújo ${ }^{2}{ }^{\circ}$, Juliana Pereira Lyon $^{30}$ \\ ${ }^{I}$ Departamento de Zootecnia, Universidade Federal de São João del-Rei, São João del-Rei, Minas Gerais, Brasil. \\ ${ }^{2}$ Instituto Federal de Educação, Ciências e Tecnologia de Tocantins, Colinas dos Tocantins, Tocantins (TO), Brasil. \\ ${ }^{3}$ Departamento de Ciências Naturais, Universidade Federal de São João del-Rei, São João del-Rei, Minas Gerais, Brasil. \\ Autor para correspondência, E-mail: leonardomarmo@gmail.com
}

\begin{abstract}
Resumo. O abomaso representa o "estômago verdadeiro" dos ruminantes e apresenta um $\mathrm{pH}$ significativamente ácido, o qual é decisivo para os processos químicos que ocorrem com sais minerais. De fato, o valor de $\mathrm{pH}$ do meio químico influencia decisivamente a precipitação ou solubilização de íons assim como sua complexação, os quais são fatores determinantes para a absorção dos íons no trato gastrintestinal (TGI) das referidas espécies animais. No entanto, nem sempre a relevância do meio ácido na formação de complexos metálicos e/ou compostos de coordenação são devidamente explicados e enfatizados na literatura focada em nutrição animal. O entendimento da relevância da complexação de cátions por moléculas e macromoléculas presentes na digesta bem como a decisiva influência do meio ácido nesse processo constituem aspectos fundamentais para a busca de estratégias nutricionais mais eficientes para uma maior absorção dos sais minerais intrínsecos aos alimentos ingeridos ou adicionados na dieta como um todo. Assim sendo, a abordagem ora apresentada constitui importante pré-requisito para viabilizar novas dietas e/ou protocolos de manejo visando a uma menor perda de sais minerais na matéria fecal. A relevância bioquímica e fisiológica dos diversos íons presentes em sais minerais e seu grande impacto no custo da dieta dos animais denotam o real value de um maior estudo e foco para essa área por parte de nutricionistas animais e demais pesquisadores de áreas afins.
\end{abstract}

Palavras chaves: sais minerais; abomaso; complexos metálicos

\section{The decisive role of the abomasal acidic medium in the mineral absorption in gastrointestinal tract of ruminants}

Abstract. The abomasum represents the "true stomach" of the ruminants and presents a significantly acid $\mathrm{pH}$, which is decisive to the chemical processes that occur with mineral salts. Indeed, the $\mathrm{pH}$ value of the chemical medium affects decisively the precipitation or solubilization of ions as well as its complexation, which are determinant factors to the ionic absorption in the gastrointestinal tract (GIT) of the respective animal species. However, the importance of the acid medium to the metallic complexes and/or coordination compounds is not always adequately explained and emphasized in the literature focused on animal nutrition. The understanding of the relevance of the cations complexation for molecules and macromolecules present in the digesta as well as the decisive influence of the acid medium in this process constitutes fundamental aspects to the search of more efficient nutritional strategies to a higher absorption of the mineral salts that are intrinsic in the food eaten or added in the complete diet. In this way, the present approach constitutes relevant pre-requisite to viabilized new diets and/or protocols of management aiming to a lower mineral salt's loss in the fecal matter. The biochemical and physiological relevance of 
several ions in the mineral salts and its great impact in the cost of the animal diet denotes the real value of a greater study and focus to this area by animal nutritionists and other researchers of similar areas.

Keywords: mineral salts, abomasum, metallic complexes

\title{
El papel decisivo del medio ácido abomasal em la absorción de minerales em el tracto gastrointestinal de los rumiantes
}

\begin{abstract}
Resumen. El abomaso representa el "verdadero estómago" de los rumiantes y tiene un $\mathrm{pH}$ significativamente ácido, que es decisivo para los procesos químicos que ocurren con las sales minerales. De hecho, el valor del pH del medio químico influye decisivamente en la precipitación o solubilización de los iones, así como en su compleción, que son factores determinantes para la absorción de iones en el tracto gastrointestinal (TGI) de las especies animales referidas. Sin embargo, la relevancia del medio ácido en la formación de complejos metálicos y / o compuestos de coordinación no siempre se explica y enfatiza adecuadamente en la literatura centrada en la nutrición animal. Comprender la relevancia de la compleción de cationes por las moléculas y macromoléculas presentes en la digesta, así como la influencia decisiva del medio ácido en este proceso, son aspectos fundamentales en la búsqueda de estrategias nutricionales más eficientes para una mayor absorción de sales minerales intrínsecas a los alimentos ingeridos o agregados en la dieta. dieta en su conjunto. Por lo tanto, el enfoque presentado aquí es un prerrequisito importante para permitir nuevas dietas y / o protocolos de manejo que tengan como objetivo una menor pérdida de sales minerales en la materia fecal. La relevancia bioquímica y fisiológica de los diversos iones presentes en las sales minerales y su gran impacto en el costo de la dieta de los animales denota el valor real de un estudio más amplio y se centra en esta área por nutricionistas de animales y otros investigadores de áreas similares.
\end{abstract}

Palabras clave: sales minerales, abomaso, complejos metálicos

\section{Introdução}

A dissociação iônica de sais minerais está diretamente relacionada ao $\mathrm{pH}$ do meio químico no qual os minerais se encontram. Como consequência, no meio biológico, a absorção de minerais depende fundamentalmente do $\mathrm{pH}$ do meio químico associado aos respectivos sais minerais. Isso ocorre, pois a dissociação dos íons e o fato dessas partículas estarem ou não associadas a outras espécies químicas afeta, substancialmente, a possibilidade de os íons conseguirem uma permeação através da bicamada fosfolipídica das membranas celulares e, portanto, sua absorção na corrente sanguínea. De fato, um dos aspectos decisivos para a absorção de minerais, e que depende diretamente do $\mathrm{pH}$ do meio, é a complexação de cátions metálicos pelas biomoléculas presentes no bolo alimentar (com os ânions, originados dos sais minerais que liberaram os cátions, interagindo ionicamente como pares iônicos) (Moreira et al., 2019).

Nesse contexto, o abomaso apresenta um papel decisivo para a absorção mineral no trato gastrintestinal (TGI) de ruminantes. O abomaso, conhecido como "estômago verdadeiro", representa um meio muito ácido que difere drasticamente dos outros compartimentos do TGI de ruminantes. O abomaso como estômago verdadeiro possui suco gástrico, o qual contém ácido clorídrico $(\mathrm{HCl})$, ácido forte (ácido que é considerado aproximadamente cem por cento ionizado em meio aquoso) que faz com que o meio apresente um $\mathrm{pH}$ altamente ácido, chegando até aproximadamente 2, na escala de $\mathrm{pH}$ em meio aquoso.

Após a formação de diversas complexações metálicas (compostos de coordenação formados ao redor dos cátions liberados na dissolução dos sais minerais, ou seja, tendo os cátions metálicos como centros de coordenação) (Basolo \& Johnson, 1980; Farias, 2005) pela complexação de cátions metálicos por meio de vários sítios doadores presentes proteínas e fibras (que atuam, portanto, como bases de Lewis, ou seja, sítios que compartilham seus pares eletrônicos livres com ácidos de Lewis, como é o caso da atuação dos cátions metálicos nesse contexto) no rúmen, uma série de processos tende a ocorrer (Moreira et al., 2013). De fato, a chegada ao abomaso e o impacto da acidez do ambiente promove o ataque dos 
prótons $\left(\mathrm{H}^{+}\right)$em direção aos sítios de pares eletrônicos (sítios básicos de Lewis), dissociando os complexos. Portanto, a dissolução dos compostos de coordenação libera fibras, proteínas, e outras biomoléculas das ligações com os cátions (e seus respectivos pares iônicos, isto é, os ânions), fazendo com que os cátions fiquem livres no meio abomasal, facilitando, com essa liberação, a disponibilidade para contatos ótimos na parede do TGI e subsequente absorção. De fato, os cátions metálicos no TGI constituem um pontos-chaves mais decisivos para a organização de estruturas relativamente estáveis que afetam a absorção de vários nutrientes, ou seja, os cátions metálicos dirigem um dos mais importantes mecanismos que afetam o grau de absorção da matéria mineral (Johnson et al., 2005).

No caso da solubilização de hidróxidos pouco solúveis, algo parecido aconteceria, ou seja, com a acidificação e neutralização das bases, os cátions seriam liberados somente no abomaso, evitando que os mesmos fossem complexados no rúmen e, assim, aumentando a quantidade de cátions livres no abomaso. De fato, o abomaso consiste em um compartimento do TGI no qual o ambiente químico ácido limita (apesar de não impedir totalmente) o sequestro dos cátions pelas fibras e proteínas, uma vez que significativa parte dos pares de elétrons dessas biomoléculas estariam protonados.

Por conseguinte, hidróxidos que sejam menos solúveis podem liberar os cátions para serem dissolvidos a partir da reação ácido-base que ocorre em meio ácido (Huheey et al., 2006; Shriver et al., 2006). Nesse caso, em se tratando de meio químico dentro do ambiente biológico (TGI), a acidez corresponde às condições intrínsecas ao meio abomasal.

\section{Antes de abomaso}

No caso dos ruminantes, o rúmen é decisivo na formação de compostos de coordenação formados por cátions como centros de coordenação e de sítios básicos de Lewis que são encontrados em fibras, proteínas, entre outras biomoléculas, como ligantes. A construção do chamado "cluster" faz com que a rede das fibras se torne mais compacta, dificultando o acesso da enzima celulase a grande número de macromoléculas celulósicas e limitando a taxa de quebra de fibras ligno-celulósicas.

\section{No abomaso}

A variação de $\mathrm{pH}$ é decisiva para o processo de formação de ligações e quebra de ligações que viabiliza e degrada, respectivamente, os complexos metálicos. Nesse contexto, o papel do abomaso é central nessa transição, uma vez que o bolo alimentar advinha do meio ruminal (valores de $\mathrm{pH}$ entre 67 aproximadamente), para $\mathrm{pH} 2$ (com posterior alcalinização após a saída desse compartimento). A chamada "cinética digestiva" depende fortemente da variação de $\mathrm{pH}$, que influencia a formação de complexos, a qual está profundamente relacionada a perda de cinzas. Essa perda de sais minerais também depende da perda de outros nutrientes, tais como fibras e proteínas.

\section{Após o abomaso}

Com a digesta deixando o abomaso, o ambiente químico em torno do bolo alimentar vai ficando menos ácido, portanto, muitos sítios protonados iniciam um processo de desprotonação, o que significa que mais sítios básicos de Lewis estarão disponíveis para atuar como ligantes para cátions, ou seja, para a formação de compostos de coordenação. De fato, o valor de $\mathrm{pH}$ do intestino vai sendo alcalinizado até aproximadamente o valor em torno de 8.0.

O aumento da excreção de sais minerais também tende a aumentar a excreção de fibras e outros nutrientes que podem estar associados ao "cluster". A presença de macromoléculas biológicas como carboidratos fibrosos e proteínas favorece a formação de uma rede de ligações covalentes coordenadas muito numerosas, as quais tendem a carrear outras moléculas, íons e espécies químicas, aumentando a perda de nutrientes pela matéria fecal.

\section{O papel de hidróxidos pouco solúveis}

Hidróxidos pouco solúveis permanecem, em grande parte, na forma de sólidos não ionizados, o que limita em uma percentagem relativamente pequena a liberação dos cátions metálicos livres em solução, 
ou seja, solubilizados pelo solvente aquoso presente no TGI. Portanto, a ingestão, por parte dos ruminantes, de hidróxidos pouco solúveis limita a liberação no seio da solução de cátions métalicos livres e acessíveis a agentes coordenantes presentes na digesta. Por conseguinte, tais bases de Arrhenius pouco solúveis fazem com que a faixa de extensão do TGI no qual os cátions metálicos estarão livres para serem "sequestradas" por bases de Lewis que atuam como agentes coordenantes ("ligantes") desses cátions será muito mais reduzida em comparação com a ingestão de sais metálicos comuns. Uma limitação semelhante ocorre com a ingestão de complexos metálicos quelatados. O abomaso desenvolve um papel-chave para a absorção de cátions oriundos de hidróxidos pouco solúveis.

\section{O papel dos complexos metálicos quelatados e macrocíclicos}

A ingestão de sais metálicos na forma de complexos metálicos, sobretudo na forma de compostos de coordenação quelatados e/ou quelatados macrocíclicos, também desenvolve um papel de mantenedor dos cátions metálicos "protegidos" de um maior ataque por bases de Lewis, o qual pode gerar o "sequestro" das espécies catiônicas (Lindoy, 1990). De fato, como já foi discutido em trabalhos anteriores, a complexação apresenta um papel-chave para limitar a absorção de sais minerais, afetando até mesmo a absorção de outros nutrientes que, eventualmente, estejam ligados a esses sais minerais (Moreira et al., 2013).

O abomaso, assim como ocorre no caso dos hidróxidos pouco solúveis, também desenvolve uma atuação decisiva para a absorção em maior ou menor grau de cátions ("centros de coordenação") assim como os próprios ânions, os quais podem estar constituindo os complexos seja como ligantes, seja como contra-íons (também chamados pares iônicos). De fato, a acidez do abomaso é decisiva para que os componentes do complexo metálico (centro de coordenação, ligantes e contra-íons) sejam separados e, a partir daí, atuem, quimicamente, de forma totalmente independente do ponto de vista da reatividade química de cada espécie.

\section{A influência de outros fatores}

Fatores como o volume de saliva também influenciam nesse processo, uma vez que o volume de solvente aquoso inevitavelmente afetará a concentração hidrogeniônica, isto é, o valor de pH do meio químico em questão, assim como a concentração dos nutrientes e biomoléculas geradas endogenamente. Além disso, o volume de saliva também influenciará a taxa de passagem da digesta, afetando, portanto, o tempo que determinado conteúdo de alimentos ficará em cada compartimento do TGI do animal. O intervalo de tempo em cada compartimento afeta o processo de absorção dos sais minerais, pois está relacionado ao deslocamento de vários equilíbrios químicos coexistentes em cada meio químico e à liberdade de ação química, ou seja, a atividade de cada íon.

É digno de nota que fatores como o volume de sucos digestivos podem influenciar na composição química de cada meio químico, em cada compartimento do TGI, o que também influenciará nos processos químicos que ocorrerem em maior ou menor grau dependendo da concentração e do tempo de contato, pois tais fatores influenciam a probabilidade de contato ótimo entre as espécies reagentes de um determinado ambiente químico.

\section{Correlações entre a influência do abomaso em ruminantes e a influência do estômago em não- ruminantes}

A influência do meio ácido é decisiva para a formação de complexos metálicos assim como para a precipitação/solubilização de sais poucos solúveis e para a solubilização de hidróxidos pouco solúveis. Portanto, uma acidez como aquela encontrada no abomaso, que pode girar em torno de $\mathrm{pH} 2$ representa um papel decisivo para os processos químicos e bioquímicos a que estão suscetíveis os íons minerais, cátions e ânions, inclusive a própria absorção.

No caso de não-ruminantes, o estômago representa esse meio no qual o pH ácido exerce significativa função na determinação na condição química de cátions e ânions. Por conseguinte, muito do que se discute nesse sentido para ruminantes poderia ser aplicado, até certo ponto, para alguns não ruminantes. No entanto, algumas diferenças devem ser destacadas, pois, no caso dos ruminantes, a passagem pelo 
ambiente ruminal e a quebra da celulose no rúmen fazem com que muitos complexos metálicos já estejam previamente formados, quando chegam ao meio ácido do abomaso. Essa complexação anterior à chegada ao $\mathrm{pH}$ mais ácido tende a ser bem menor no caso de não-ruminantes, o que implica que grande parte da complexação tenderá a acontecer já no intestino, quando o $\mathrm{pH}$ não estiver tão ácido. Pode ocorrer, por conseguinte, que grande parte dos íons já tenham sido absorvidos.

No caso de não-ruminantes, um fator que tende, a priori, a favorecer a complexação seria a ausência da celulase e, portanto, da digestão química da celulose, o que mantém as fibras em condições de formarem ligações covalentes coordenadas com cátions e contatos iônicos com os ânions em toda a extensão do TGI. Ainda assim, os vários compartimentos do TGI de ruminantes e o período da digesta no rúmen tende a ser decisivos para a formação de compostos de coordenação que podem ser mais ou menos estáveis no TGI, dependendo de vários fatores, tais como relação carga-raio do cátion, afinidade química entre cátions e sítios doadores de pares de elétrons, composição química da dieta etc. De qualquer maneira, o meio ácido atuará de forma decisiva para a separação dos componentes dos complexos metálicos (centro de coordenação; ligantes; e contra-íons ou pares iônicos) em maior ou menor grau, o que será decisivo em relação ao nível de absorção mineral no TGI.

É digno de nota que, após o abomaso, a digesta atinge o início do intestino delgado, que é o órgão da absorção por excelência e onde o pH ainda estará significativamente ácido (apesar de menos ácido do que no abomaso). Logo, todo o processo de "desconstrução" dos complexos metálicos efetuado no abomaso em função da grande acidez do meio repercutirá diretamente na predisposição à absorção dos sais minerais no início do intestino delgado.

Subsequentemente, conforme a digesta vai se deslocando no TGI, o pH irá aumentar lenta e gradualmente, gerando desprotonação de vários sítios doadores de pares de elétrons das diversas moléculas e macromoléculas presentes. Assim, essa paulatina alcalinização do entorno dos compostos químicos presentes no bolo alimentar tende a favorecer, cada vez mais, uma nova complexação dos cátions remanescentes, ou seja, daqueles cátions que ainda não tenham sido absorvidos.

\section{Conclusões}

A compreensão do papel do abomaso e do $\mathrm{pH}$ ácido que esse compartimento do trato gastrintestinal (TGI) apresenta na absorção de sais minerais em ruminantes é pré-requisito fundamental para a elaboração de melhores estratégias nutricionais visando a um melhor aproveitamento dos sais minerais ingeridos na alimentação. Nesse contexto, a influência do $\mathrm{pH}$ no processo de complexação de cátions metálicos, com o concomitante acompanhamento de ânions como contra-íons, durante o deslocamento dos alimentos no TGI, é requisito conceitual decisivo para determinar a absorção de minerais em maior ou menor grau em cada um dos diferentes setores do TGI, os quais apresentam diferentes faixas de $\mathrm{pH}$. De fato, a influência do pH ácido na complexação de sais minerais é importante para diferentes espécies animais, abrangendo ruminantes e não-ruminantes. Além disso, a complexação afeta substancialmente a absorção dos sais minerais e, dessa forma, altera o grau de absorção de sais minerais ingeridos no processo de alimentação. É muito importante frisar que quanto mais cátions metálicos forem complexados e, dessa forma, encaminhados para uma "perda nutricional"(em função da não-absorção) pela excreção fecal, mais perda será, igualmente, acarretada no que se refere a outros tipos de nutrientes que se ligam aos cátions metálicos e podem ser eliminados do TGI. Portanto, quanto maior for a perda de sais minerais, maior tende a ser a perda de carboidratos, proteínas e outros nutrientes. Estudos focados em engenharia florestal e áreas interdisciplinares e correlacionadas à química e à bioquímica e às ciências agrárias em geral envolvendo complexos metálicos tem demonstrado que diferentes amostras de biomassa, tais como massas celulósicas, podem reter grande quantidade de cátions metálicos, incluindo cátions metálicos, os quais podem ser oriundos de metais alcalinos, metais alcalinos terrosos, metais de transição e até mesmo metais de transição interna. Considerando que os sais minerais constituem importantíssimos nutrientes para o bem-estar bioquímico e fisiológico das mais distintas espécies animais, além de representarem relevante custo para alimentação, o entendimento tanto da complexação propriamente considerada como da influência do meio ácido que ocorre nos ruminantes no abomaso constitui estudo fundamental para a definição de melhores estratégias nutricionais, tanto no que se refere aos nutrientes selecionados para a alimentação como no que diz respeito ao manejo das culturas de uma forma geral. 


\section{Referências bibliográficas}

Basolo, F., \& Johnson, R. (1980). Química de los compuestos de coodinación - La química de los complejos metalicos. Editorial Reverté, S.A.

Farias, R. F. (2005). Química de Coordenação - Fundamentos e atualidades. Editora Átomo.

Huheey, J. E., Keiter, E. A., Keiter, R. L., \& Medhi, O. K. (2006). Inorganic chemistry: principles of structure and reactivity. Pearson Education India.

Johnson, T. R., Eicher, S. D., \& McKee, C. A. (2005). Animal feed compositions and methods of using the same. Google Patents.

Lindoy, L. F. (1990). The chemistry of macrocyclic ligand complexes. Cambridge University Press.

Moreira, L. M., Leonel, F. de P., Vieira, R. A. M., \& Pereira, J. C. (2013). A new approach about the digestion of fibers by ruminants. Revista Brasileira de Saúde e Produção Animal, 14(2), 382-395. DOI: http://dx.doi.org/10.1590/S1519-99402013000200008

Moreira, L. M., Teixeira, A. O., Machado, H. V. N., Araújo, R. P., \& Lyon, J. P. (2019). Complexos metálicos no trato gastrintestinal, como um tópico relevante para a compreensão da nutrição mineral: Revisão. PUBVET, 13(10), 1-6. DOI: https://doi.org/10.31533/pubvet.v13n10a432.1-6

Pereira, A. A., Martins, G. F., Antunes, P. A., Conrrado, R., Pasquini, D., Job, A. E., Curvelo, A. A. S., Ferreira, M., Riul, A., \& Constantino, C. J. L. (2007). Lignin from sugar cane bagasse: extraction, fabrication of nanostructured films, and application. Langmuir, 23(12), 6652-6659. DOI: https://doi.org/10.1021/la063582s

Shriver, D. F., Atkins, P. W., Overton, T. L., Rourke, J. P., Weller, M. T., \& Armstrong, F. A. (2006). Inorganic Chemistry. Oxford University Press.

Van Soest, P. J., Robertson, J. B., \& Lewis, B. A. (1991). Methods for dietary fiber, neutral detergent fiber, and nonstarch polysaccharides in relation to animal nutrition. Journal of Dairy Science, 74(10), 3583-3597. https://doi.org/http://dx.doi.org/10.3168/jds.S0022-0302(91)78551-2.

Recebido: 3 de fevereiro, 2020 . Aprovado: 15 de março, 2020. Disponível online: 14 julho, 2020.
Licenciamento: Este artigo é publicado na modalidade Acesso Aberto sob a licença Creative Commons Atribuição 4.0 (CC-BY 4.0), a qual permite uso irrestrito, distribuição, reprodução em qualquer meio, desde que o autor e a fonte sejam devidamente creditados 